\title{
A importância do enfermeiro no estímulo cognitivo e de memória em pessoas idosas: revisão integrativa
}

The importance of nurses in cognitive and memory stimulation in the elderly: an integrative review

La importancia de las enfermeras en la estimulación cognitiva y de memoria en los ancianos: una revisión integradora

Recebido: 02/05/2020 | Revisado: 04/05/2020 | Aceito: 06/05/2020 | Publicado: 12/05/2020

\section{Lucas de Almeida Abrahão}

ORCID: https://orcid.org/0000-0002-2731-0047

Universidade Federal Fluminense, Brasil

E-mail: lucascs200@ hotmail.com

\section{Alessandra Conceição Leite Funchal Camacho}

ORCID: https://orcid.org/0000-0001-6600-6630

Universidade Federal Fluminense, Brasil

E-mail: cicacamacho@gmail.com.br

\section{Resumo}

Trata-se de uma revisão integrativa, cujo objetivo é analisar as produções científicas acerca do papel do enfermeiro no estímulo cognitivo e de memória em pessoas idosas, a partir dos artigos levantados nas bases de dados online. Foram selecionadas para o estudo nove publicações encontradas nas bases de dados científicas nacionais Lilacs, Medline, BDENF, PUBMED e SCOPUS. Os descritores utilizados foram: Cognição; Enfermagem; Idoso; Memória, sendo selecionados 09 artigos. Na discussão foram abordados: $\mathrm{O}$ estímulo cognitivo e de memória em pessoa idosa sem Diagnóstico de Síndromes Demenciais e o estímulo cognitivo e de memória em pessoa idosa com Diagnóstico de Síndromes Demenciais. Conclui-se que a importância do enfermeiro no estímulo cognitivo e de memória da pessoa idosa é crucial para a qualidade de vida da população idosa, buscando novas estratégias e tecnologias para a manutenção da cognitiva e da memória em idosos saudáveis, dessa forma, retardando o avanço das síndromes demenciais, principalmente a Doença de Alzheimer. 
Palavras-chave: Cognição; Enfermagem; Idoso; Memória.

\begin{abstract}
It is an integrative review, whose objective is to analyze scientific productions about the role of nurses in cognitive and memory stimulation in elderly people, based on the articles collected in the online databases. Nine publications found in the national scientific databases Lilacs, Medline, BDENF, PUBMED and SCOPUS were selected for the study. The descriptors used were: Cognition; Nursing; Old man; Memory, with 09 articles selected. In the discussion were addressed: The cognitive and memory stimulus in an elderly person without Diagnosis of Dementia Syndromes and the cognitive and memory stimulus in an elderly person with Diagnosis of Dementia Syndromes. It is concluded that the importance of the nurse in the cognitive and memory stimulation of the elderly is crucial for the quality of life of the elderly population, seeking new strategies and technologies for the maintenance of cognitive and memory in healthy elderly people, thus, delaying the advancement of dementia syndromes, especially Alzheimer's disease.
\end{abstract}

Keywords: Cognition; Nursing; Elderly; Memory.

\title{
Resumen
}

Es una revisión, cuyo objetivo es analizar las producciones científicas sobre el papel de las enfermeras en la estimulación cognitiva y de memoria en las personas mayores, en base a los artículos recopilados en las bases de datos en línea. Nueve publicaciones encontradas en las bases de datos científicas nacionales Lilacs, Medline, BDENF, PUBMED y SCOPUS fueron seleccionadas para el estudio. Los descriptores utilizados fueron: Cognición; Enfermería; Ancianos Memoria, sendo selecionados 09 artigos. En la discusión se abordaron: el estímulo cognitivo y de memoria en una persona mayor sin diagnóstico de síndromes de demencia y el estímulo cognitivo y de memoria en una persona mayor con diagnóstico de síndromes de demencia. Se concluye que la importancia de las enfermeras en la estimulación cognitiva y de memoria de los ancianos es crucial para la calidad de vida de la población anciana, buscando nuevas estrategias y tecnologías para mantener la inteligencia cognitiva y la memoria en personas mayores sanas y retrasar el progreso de síndromes de demencia, especialmente la enfermedad de Alzheimer.

Palabras clave: Cognición; Enfermería; Anciano; Memoria. 


\section{Introdução}

Segundo a Organização Mundial de Saúde (2014), o número de indivíduos com idade maior ou igual a 60 anos chegará à 2 bilhões de pessoas até 2050, ou seja, um quinto da população mundial atualmente. Dados coletados pelo Ministério da Saúde (MS) afirmam que em 2016, o Brasil tinha a quinta maior população idosa do mundo, sendo que em 2030, o número de idosos irá ultrapassar o total de crianças entre zero e 14 anos de idade (OMS, 2014).

No entanto, a Pesquisa Nacional por Amostra de Domicílios Contínua (IBGE, 2018), apresenta a tendência de envelhecimento no Brasil, a qual se manteve nos últimos anos, elevando 4,8 milhões de idosos desde 2012, e ultrapassando a marca dos 30,2 milhões em 2017. Esses 5 anos corresponderam a um crescimento de $18 \%$ da população idosa e cada vez mais vem se destacando entre as pirâmides etárias brasileiras. As mulheres são a maioria da população idosa, com 16,9 milhões (56\%), enquanto os homens de idade avançada representam 13,3 milhões (44\%).

Entre 2012 e 2017, o número de idosos cresceu em todos os estados do Brasil, principalmente no estado do Rio de Janeiro e Rio Grande do Sul, ambas alcançando 18,6\% de suas populações dentro do grupo de 60 anos ou mais (IBGE, 2018).

É importante salientar que a maior parte das patologias crônicas que acometem o idoso tem seu principal fator de risco própria idade. Sendo assim, não é o aparecimento ou não da doença que vai definir a qualidade de vida, mas a capacidade que o indivíduo terá para seguir a própria vida de maneira independente e autônoma.

Essa busca por um envelhecimento com maior qualidade se mostra evidente o aumento da procura de atividades que possibilitam o bem-estar e que, consequentemente, estejam relacionadas ao cuidado da saúde física e mental. A motivação para esta busca de atividades saudáveis pode estar relacionada ao receio do idoso de adquirir doenças que afetam a sua autonomia e independência.

Uma das mudanças mais comuns no processo de envelhecimento é a perda de memória, seja proveniente do processo de envelhecimento normal ou relacionado a alguma doença, e que pode causar agravos no desempenho de atividades do cotidiano e também na percepção de autoeficácia e autoestima influenciando na qualidade de vida do indivíduo idoso (Morando, Schmitt \& Ferreira, 2017, p. 354).

As informações e as orientações sobre o autocuidado e a prevenção de patologias incapacitantes do idoso são, em sua maioria, realizadas pelos profissionais de saúde, 
principalmente o enfermeiro. Porém, isso não impede que familiares e amigos possam ajudar nos cuidados com o idoso. O enfermeiro tem o dever de orientar, cooperar e dar suporte para que o indivíduo possa realizar seu autocuidado de maneira eficaz, priorizando seu bem-estar, autonomia e independência (Ferreira et al, 2012).

Diante dessas considerações o objetivo deste estudo é analisar as produções científicas acerca do papel do enfermeiro no estímulo cognitivo e de memória em pessoas idosas.

$\mathrm{O}$ estudo presente mostra-se relevante a partir do momento que o número de idosos vem aumentando significantemente no Brasil e no mundo, reforçando as necessidades do cuidado com a memória e cognição da pessoa idosa, que afeta diretamente na qualidade de vida dessa população.

Do ponto de vista científico, em pesquisa prévia em bases de dados de literatura científica, ressalta-se a necessidade de uma assistência de enfermagem qualificada. Com isso, o presente estudo busca, além de, enriquecer o notório campo da pesquisa gerontológica em enfermagem, instigar a investigação sobre tal tema.

\section{Metodologia}

Este estudo caracteriza-se como uma revisão integrativa, (Soares et al, 2014), a qual é definida como um tipo de revisão da literatura que reúne achados de estudos desenvolvidos por meio de diferentes metodologias, possibilitando aos revisores sintetizar resultados sem alterar a filiação epistemológica dos estudos empíricos envolvidos. Para que esse método se concretize de maneira coerente, livre de alienações epistemológicas, a revisão integrativa requer que os revisores operem à análise e a síntese das informações primárias de forma sistemática e rigorosa.

Dessa forma, essa metodologia percorreu as seguintes etapas: 1. Formulação do problema e objetivos da revisão, 2. Estabelecimento de critérios de inclusão e exclusão de artigos, 3. Definição das informações a serem extraídas dos artigos selecionados e avaliação de dados, 4. Análise e interpretação dos dados, e 5. Discussão e apresentação dos resultados (Souza, Silva \& Carvalho, 2010).

Buscando entender a primeira etapa da revisão foi estabelecido a seguinte questão norteadora: Qual o papel do estímulo cognitivo e memória para a pessoa idosa na literatura de enfermagem?

Logo em seguida, foi realizada a busca nos seguintes bancos de dados da Biblioteca Virtual de Saúde (BVS): Lilacs (Literatura Latino Americana e do Caribe em Ciências da 
Saúde), Medline (Biblioteca Nacional de Medicina) e BDENF (Base de Dados de Enfermagem), e nas bases de dados internacionais PUBMED (Medical Published - service of the U.S. National Library of Medicine) e SCOPUS.

A escolha dessas bases de dados foi estritamente por sua relevância na área da enfermagem e de saúde em termos de publicações científicas atualizadas e fornecimento de evidências significativas para o desenvolvimento do conhecimento.

Os descritores, utilizando operador Booleano AND, foram: Cognição; Enfermagem; Idoso; Memória. Nos idiomas português, inglês e espanhol. O período de coleta de dados foi durante o mês de maio de 2019.

Os critérios de inclusão utilizados foram: artigos que respondem as questões de pesquisa estabelecidas pelo estudo publicado na integra online, artigos publicados em português, inglês e espanhol; Artigos publicados na íntegra que destacassem as temáticas "Cognição", "Enfermagem", "Idoso" e "Memória", estudos realizados com humanos de ambos os sexos e etnias e artigos publicados nos referidos bancos de dados nos anos de 2009 a 2019. Os critérios de exclusão foram artigos repetidos.

Para organização do conteúdo obtido foi elaborado um quadro contendo os seguintes itens: ano, base de dados, tipos de publicação e abordagem metodológica, essência do conteúdo/produção do conhecimento e recomendação dos autores.

A seleção das bibliografias potenciais se baseou nos artigos fundamentais a responder os pré-requisitos da pesquisa. Posteriormente, realizou-se a impressão e leitura dos artigos na íntegra com a finalidade de estabelecer relações textuais, contextuais e intertextuais, visando o surgimento de categorias que permitiram a discussão dos objetivos propostos nesse estudo em questão.

Este estudo se enquadra no eixo 12 (Saúde do Idoso), subitem 12.5 (Análise da Gestão e das Práticas das Equipes no cuidado às especificidades em saúde da pessoa idosa) da Agenda nacional de prioridades de pesquisa em saúde - ANPPS (Ministério da Saúde, 2018).

\section{Resultados}

Na base de dados da Biblioteca Virtual em Saúde (BVS) foram encontrados 42 artigos através da combinação dos descritores: Cognição AND Enfermagem AND Idoso AND Memória. Com a aplicação dos critérios de inclusão e exclusão restaram 2 artigos.

Na LILACS foram selecionados 1 dos 10 artigos encontrados após a aplicação dos critérios de inclusão. Na MEDLINE foram encontrados 27 artigos, e logo após o uso dos 
Research, Society and Development, v. 9, n. 7, e306974180, 2020

(CC BY 4.0) | ISSN 2525-3409 | DOI: http://dx.doi.org/10.33448/rsd-v9i7.4180

critérios, foram selecionados 1 artigo. Na BDENF nenhum dos 5 artigos encontrados foram selecionados a partir dos critérios de inclusão.

Na base de dados PUBMED foram encontrados 597 artigos através da combinação dos descritores: Cognition AND Nursing AND Elderly AND Memory. A partir da aplicação dos critérios de inclusão e exclusão restaram 123 artigos, após leitura fluente foram selecionados 5 artigos.

Na base de dados SCOPUS foram encontrados 163 artigos após a aplicação dos descritores: Cognition AND Nursing AND Elderly AND Memory. A partir da aplicação dos critérios de inclusão e exclusão restaram 5 publicações, após a leitura fluente foram selecionados 2 artigos.

Segue abaixo o Fluxograma sobre a seleção dos artigos:

Figura 1: Fluxograma sobre a seleção dos artigos (Niterói, 2020).

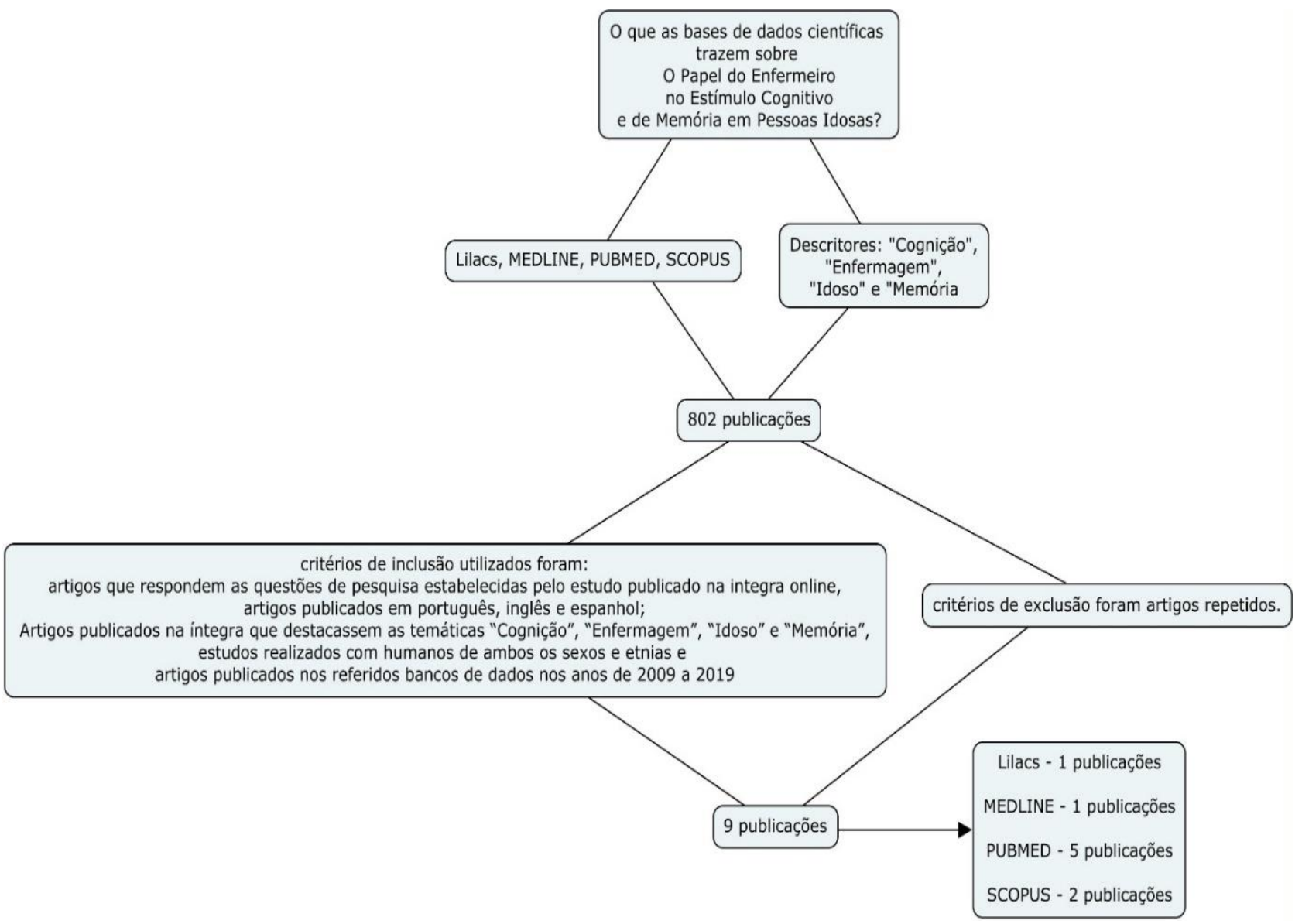

Fonte: Autores (2020) 
Research, Society and Development, v. 9, n. 7, e306974180, 2020

(CC BY 4.0) | ISSN 2525-3409 | DOI: http://dx.doi.org/10.33448/rsd-v9i7.4180

Quadro 1: Quantitativo de artigos selecionados por ano (Niterói, 2020).

\begin{tabular}{|c|c|c|}
\hline Ano & Número & \% \\
\hline 2009 & 1 & 11 \\
\hline 2010 & 1 & 11 \\
\hline 2012 & 1 & 11 \\
\hline 2013 & 2 & 22 \\
\hline 2015 & 1 & 11 \\
\hline 2017 & 2 & 22 \\
\hline 2018 & 1 & 11 \\
\hline Total & 9 & 100 \\
\hline
\end{tabular}

Fonte: Autores (2020)

Foram pesquisados artigos dos últimos 10 anos (2009-2019), onde verifica-se ausência de obras referente ao tema nos anos 2011, 2014, 2016 e 2019. Evidencia-se 2 artigos no ano de 2013 e 2 artigos no ano de 2017, sendo que cada um representou 22\% das publicações, totalizando 44\%. Os anos de 2009, 2010, 2012, 2013, 2015, 2016 e 2018 tiveram o menor índice de artigos, com $11 \%$ cada.

Em relação às abordagens metodológicas encontradas entre os artigos, destacaram-se os estudos clínicos randomizados com 33\% (3 artigos). No entanto, outras abordagens como: Estudo de Caso, Estudo Transversal, Análise Multivariada, Estudo Longitudinal, Estudo Quasi-Experimental e Estudo de Coorte Prospectivo tiveram apenas 1 artigo cada, o equivalente a $11 \%$.

As obras foram encontradas em publicações de 9 revistas, sendo que não houveram repetições de revistas entre os artigos, estabelecendo um percentual de $11 \%$ cada. 
Research, Society and Development, v. 9, n. 7, e306974180, 2020

(CC BY 4.0) | ISSN 2525-3409 | DOI: http://dx.doi.org/10.33448/rsd-v9i7.4180

Quadro 2: Quantitativo de artigos selecionados por revista (Niterói, 2020).

\begin{tabular}{|l|c|}
\hline \multicolumn{1}{|c|}{ Revista } & \% \\
\hline Alzheimers Dement & 11 \\
\hline Arch Psychiatr Nursing & 11 \\
\hline Geriatrics and Gerontoly Internacional & 11 \\
\hline Journal of Korean Academy of Nursing & 11 \\
\hline Journal of Preventive Medicine \& Public Health & 11 \\
\hline Nursing Res. & 11 \\
\hline Res. Nursing Health & 11 \\
\hline Revista Brasileira de Enfermagem & 11 \\
\hline Revista Mineira de Enfermagem & 11 \\
\hline Total & 100 \\
\hline
\end{tabular}

Fonte: Autores (2020).

O Quadro 2 revela que não houve prevalência entre as revistas das publicações que foram selecionadas. Cada periódico apresentou apenas um artigo selecionado para a realização dessa pesquisa.

Quadro 3: Categorização das Publicações por Público (Niterói, 2020).

\begin{tabular}{|c|c|}
\hline Tipo de Participante do Estudo & $\%$ \\
\hline $\begin{array}{l}\text { Pessoa Idosa sem Diagnóstico de Síndromes } \\
\text { Demenciais }\end{array}$ & 56 \\
\hline $\begin{array}{l}\text { Pessoa Idosa com Diagnóstico de Síndromes } \\
\text { Demenciais }\end{array}$ & 44 \\
\hline Total & 100 \\
\hline
\end{tabular}

Fonte: Autores (2020).

Observa-se acima o quadro de categorização das obras selecionadas a partir do público escolhido para a realização da pesquisa.

A categoria Pessoa Idosa sem Diagnóstico de Síndromes Demenciais destacou-se em 56\% das publicações, enquanto a categoria Pessoa Idosa com Diagnóstico de Síndromes Demenciais esteve presente em $44 \%$ dos artigos.

De acordo com as publicações pesquisadas os eixos temáticos pertinentes para a discussão são: O estímulo cognitivo e de memória em pessoa idosa sem Diagnóstico de Síndromes Demenciais e o estímulo cognitivo e de memória em pessoa idosa com Diagnóstico de Síndromes Demenciais. 
Abaixo o Quadro 4 com a Síntese dos artigos selecionados:

Quadro 4: Organização do conteúdo de referências (Niterói, 2020).

\begin{tabular}{|c|c|c|c|}
\hline Ano/Título/Autor & $\begin{array}{c}\text { Tipo de Publicação } \\
\text { e Abordagem Metodológica }\end{array}$ & $\begin{array}{c}\text { Essência } \\
\text { do Conteúdo/ } \\
\text { Produção do Conhecimento }\end{array}$ & $\begin{array}{l}\text { Base } \\
\text { De } \\
\text { Dados }\end{array}$ \\
\hline $\begin{array}{lr}1-\text { Yu, F., Ryan, L. } \\
\text { H., Schaie, K. W., } \\
\text { Willis, S. } & \text { L., \& } \\
\text { Kolanowski, } & \text { A. } \\
(2009) . & \text { Factors } \\
\text { Associated } & \text { With } \\
\text { Cognition in } & \text { Adults: } \\
\text { The } & \text { Seattle } \\
\text { Longitudinal } & \text { Study. } \\
\text { Res. Nurs. Health, } \\
\text { 32(5), 540-550. }\end{array}$ & 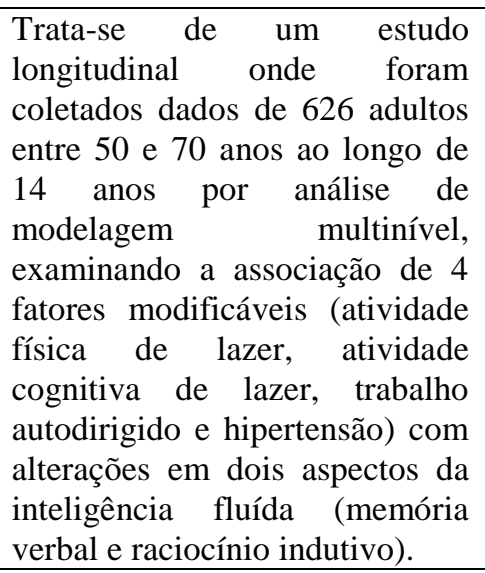 & $\begin{array}{l}\text { Através das análises a partir } \\
\text { dos dados coletados, notou-se } \\
\text { que com o passar da idade a } \\
\text { memória verbal e o raciocínio } \\
\text { indutivo vem diminuindo. As } \\
\text { análises também apontaram } \\
\text { que quanto maior o nível de } \\
\text { escolaridade, ser mulher e ter } \\
\text { uma renda maior influenciaria } \\
\text { positivamente para a memória } \\
\text { verbal e o raciocínio indutivo } \\
\text { do indivíduo. }\end{array}$ & PUBMED \\
\hline 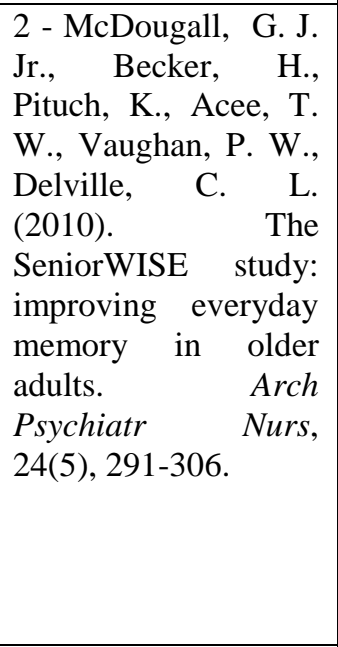 & $\begin{array}{l}\text { Estudo clínico randomizado } \\
\text { com 265 pacientes (135 no } \\
\text { treinamento de memória e } 130 \\
\text { no treinamento de promoção a } \\
\text { saúde) onde os critérios de } \\
\text { inclusão são: idade maior ou } \\
\text { igual que } 65 \text { anos, fluência em } \\
\text { inglês, algum acometimento } \\
\text { sensorial ou cognitivo e } \\
\text { disposição para participar do } \\
\text { estudo por } 24 \text { meses. O Mini- } \\
\text { Exame de Estado Mental foi } \\
\text { aplicado para descobrir } \\
\text { comprometimentos cognitivos e } \\
\text { para acompanhamento do estado } \\
\text { cognitivo ao longo do estudo. }\end{array}$ & $\begin{array}{l}\text { No início da pesquisa, os } \\
\text { indivíduos mais velhos, com } \\
\text { menor grau de escolaridade, } \\
\text { negros e hispânicos } \\
\text { apresentaram escores muito } \\
\text { baixos na memória visual. } \\
\text { Indivíduos com menor grau de } \\
\text { escolaridade, negros e } \\
\text { hispânicos também tiveram } \\
\text { escores mais baixos na } \\
\text { memória verbal. Ao final da } \\
\text { pesquisa, esses mesmo } \\
\text { indivíduos apresentaram } \\
\text { escores mais altos e menores } \\
\text { queixas sobre perda de } \\
\text { memória. }\end{array}$ & $D$ \\
\hline $\begin{array}{l}3 \text { - Mcdougall, J. R., } \\
\text { Graham J.; Mackert, } \\
\text { M. \& Becker, H. } \\
(2012) . \quad \text { Memory } \\
\text { Performance, Health } \\
\text { Literacy, rand } \\
\text { Instrumental } \\
\text { Activities of Daily } \\
\text { Living of Community } \\
\text { Residing Older } \\
\text { Adults. Nursing Res, } \\
\text { 61(1),70-75. }\end{array}$ & $\begin{array}{l}\text { Estudo clínico randomizado que } \\
\text { consistiu de } 45 \text { indivíduos, com } \\
\text { idade média de } 77 \text { anos. Foram } \\
\text { utilizados testes para determinar } \\
\text { o desempenho diário da } \\
\text { memória, o nível de } \\
\text { conhecimento em saúde e o } \\
\text { desempenho nas habilidades de } \\
\text { comunicação, habilidades } \\
\text { financeiras, habilidades de } \\
\text { compra e habilidades de } \\
\text { medicação. }\end{array}$ & $\begin{array}{l}\text { Os indivíduos do presente } \\
\text { estudo apresentaram } \\
\text { melhorias em sua capacidade } \\
\text { cognitiva, memória, } \\
\text { habilidades funcionais e uso } \\
\text { de estratégias de memória ao } \\
\text { longo da pesquisa. Nota-se } \\
\text { também que características } \\
\text { demográficas influenciaram } \\
\text { nas variações da pesquisa, por } \\
\text { exemplo, idosos tiveram } \\
\text { maiores ganhos de memória } \\
\text { visual ao longo da pesquisa. }\end{array}$ & $\mathrm{D}$ \\
\hline $\begin{array}{lr}4-\text { Kawashima, R. } \\
\text { (2013). } & \text { Mental } \\
\text { Exercises } & \text { For } \\
\text { Cognitive } & \text { Function: } \\
\text { Clinical Evidence. } \\
\text { Journal of Preventive } \\
\text { Medicine and Public } \\
\text { Health, 46(1), 22-27. }\end{array}$ & $\begin{array}{l}\text { Estudo clínico randomizado } \\
\text { dividido em dois estudos: O } \\
\text { primeiro com } 32 \text { pacientes ( } 16 \\
\text { do grupo experimental e } 16 \text { do } \\
\text { grupo de controle) que foram } \\
\text { recrutados em um lar de idosos, } \\
\text { com diagnóstico clínico de } \\
\text { demência senil tipo Alzheimer. }\end{array}$ & $\begin{array}{l}\text { Os resultados dos estudos } \\
\text { mostraram indícios positivos } \\
\text { de que um programa de } \\
\text { treinamento diário contendo } \\
\text { atividades de leitura e } \\
\text { problemas matemáticos tem } \\
\text { um efeito imediato e favorável } \\
\text { à função mental, sendo que os } \\
\text { participantes do presente }\end{array}$ & MED \\
\hline
\end{tabular}




\begin{tabular}{|c|c|c|c|}
\hline & & $\begin{array}{l}\text { estudo mantiveram essas } \\
\text { melhorias seis meses após o } \\
\text { programa de treinamento. }\end{array}$ & \\
\hline $\begin{array}{l}5 \text { - Delavande, A., } \\
\text { Hurd, M. D., } \\
\text { Martorell, P. \& } \\
\text { Langa, K. M. (2013). } \\
\text { Dementia and out-of- } \\
\text { pocket spending on } \\
\text { health care services. } \\
\text { Alzheimers Dement, } \\
\text { 9(1), 19-29. }\end{array}$ & $\begin{array}{l}\text { Trata-se de uma análise } \\
\text { multivariada baseada em dados } \\
\text { do Aging, Demographics, and } \\
\text { Memory Study que combinam } \\
\text { informações sobre: diagnósticos } \\
\text { clínicos de demência, } \\
\text { informações sobre gastos e } \\
\text { utilização de cuidados de saúde } \\
\text { e informações detalhadas sobre } \\
\text { características demográficas e } \\
\text { comorbidades que podem } \\
\text { confundir a relação entre } \\
\text { demência e gastos fora do } \\
\text { orçamento. }\end{array}$ & $\begin{array}{l}\text { Os resultados mostram que a } \\
\text { demência está associada a } \\
\text { maiores gastos médicos fora } \\
\text { do orçamento e que essa } \\
\text { ligação é estimulada } \\
\text { principalmente por gastos com } \\
\text { cuidados de enfermagem } \\
\text { domiciliar. }\end{array}$ & PUBMED \\
\hline $\begin{array}{l}6 \text { - Cruz, T. J. P., Sá, } \\
\text { S. P. C., Lindolpho, } \\
\text { M. C. \& Caldas, C. P. } \\
\text { (2015). Estimulação } \\
\text { cognitiva para idoso } \\
\text { com Doença de } \\
\text { Alzheimer realizada } \\
\text { pelo cuidador. } \\
\text { Revista Brasileira de } \\
\text { Enfermagem, 68(4), } \\
\text { 510-516. }\end{array}$ & $\begin{array}{l}\text { Trata-se de um estudo de caso, } \\
\text { de caráter descritivo e } \\
\text { abordagem qualitativa, feito } \\
\text { com cinco idosos e seus } \\
\text { respectivos cuidadores atendidos } \\
\text { no Programa intitulado "A } \\
\text { Enfermagem na Atenção a } \\
\text { Saúde do Idoso e seus } \\
\text { Cuidadores da Universidade } \\
\text { Federal Fluminense } \\
\text { (EASIC/UFF)", que realizou } \\
\text { diversas atividades coordenadas } \\
\text { pelos enfermeiros docentes da } \\
\text { UFF como: consultas de } \\
\text { enfermagem ao idoso e ao } \\
\text { cuidador, palestras para } \\
\text { orientação e informação, } \\
\text { oficinas e visitas domiciliares. }\end{array}$ & $\begin{array}{l}\text { Os resultados } r \text { encontrados } \\
\text { mostraram que, tanto idosos } \\
\text { quanto cuidadores, foram } \\
\text { beneficiados com a } \\
\text { implantação da estratégia, } \\
\text { pois colaboraram para } \\
\text { aprimorar a compreensão dos } \\
\text { cuidadores em relação à } \\
\text { importância } \\
\text { seguimento de as atividades } \\
\text { abordadas nas oficinas } \\
\text { terapêuticas. }\end{array}$ & MEDLINE \\
\hline $\begin{array}{l}7 \text { - Liang, C., Chou, } \\
\text { M., Chen, L., Wang, } \\
\text { K., Lin, S., Chen, L., } \\
\text { Lin, Y., Liu, T. \& } \\
\text { Loh, C. (2017). } \\
\text { Delaying cognitive } \\
\text { and physical decline } \\
\text { through multidomain } \\
\text { interventions for } \\
\text { residentes with mild- } \\
\text { to-moderate dementia } \\
\text { in dementia care units } \\
\text { in Taiwan: A } \\
\text { prospective cohort } \\
\text { study. Geriatr. } \\
\text { Gerontol. Int, 17(1), } \\
\text { 36-43. }\end{array}$ & $\begin{array}{l}\text { Trata-se de um estudo de coorte } \\
\text { prospectivo que verifica as } \\
\text { intervenções através de uma } \\
\text { equipe multidisciplinar, medidas } \\
\text { não-farmacológicas, avaliação e } \\
\text { intervenção geriátrica } \\
\text { abrangente e abordagem do } \\
\text { Green Channel (Equipe médica } \\
\text { que da suporte a pacientes com } \\
\text { demência). Participaram do } \\
\text { estudo } 147 \text { voluntários com } 65 \\
\text { anos ou mais. }\end{array}$ & $\begin{array}{l}\text { O estudo demonstra que as } \\
\text { intervenções de múltiplos } \\
\text { domínios desempenham } \\
\text { funções importantes na } \\
\text { melhoria cognitiva e reduzem } \\
\text { a deterioração do desempenho } \\
\text { funcional em idosos com } \\
\text { demência leve a moderada. } \\
\text { Também foi mostrado os } \\
\text { efeitos da recuperação de } \\
\text { síndromes geriátricas e a } \\
\text { redução do aparecimento de } \\
\text { novas, principalmente em } \\
\text { episódios de quedas, } \\
\text { incontinência urinária e risco } \\
\text { de desnutrição. }\end{array}$ & SCOPUS \\
\hline $\begin{array}{l}8 \text { - Kim, E. \& Suh, S. } \\
\text { (2017). Effects of a } \\
\text { Memory and Visual- } \\
\text { Motor Integration } \\
\text { Program for Older } \\
\text { Adults Based on Self- } \\
\text { Efficacy Theory. } \\
\text { Journal of Korean }\end{array}$ & $\begin{array}{l}\text { Trata-se de um estudo quase- } \\
\text { experimental, com } 62 \text { idosos } \\
\text { (grupo experimental }=30 \text {, grupo } \\
\text { controle }=32 \text { ) sem déficit } \\
\text { cognitivo ou demência } \\
\text { residentes de lares de repouso. O } \\
\text { grupo experimental participou } \\
\text { de um projeto de } 12 \text { sessões de }\end{array}$ & $\begin{array}{l}\text { Os resultados da pesquisa } \\
\text { mostraram que o projeto foi } \\
\text { eficaz para aprimorar a auto- } \\
\text { eficácia da memória, memória } \\
\text { e integração visuo-motora em } \\
\text { pessoas idosas. Os achados } \\
\text { podem influenciar em estudos } \\
\text { futuros de intervenção }\end{array}$ & SCOPUS \\
\hline
\end{tabular}




\begin{tabular}{|c|c|c|c|}
\hline $\begin{array}{l}\text { Academy of Nursing, } \\
47(3), 431-444 .\end{array}$ & $\begin{array}{l}\text { memória e integração visuo- } \\
\text { motora durante } 6 \text { semanas. }\end{array}$ & cognitiva para pessoas idosas. & \\
\hline $\begin{array}{l}9 \text { - Grden, C. R. B., } \\
\text { Ivastcheschen, T., } \\
\text { Cabral, L. P. A., } \\
\text { Reche, P. M., } \\
\text { Rodrigues, C. R. B. } \\
\text { \& Borges, P. K. O. } \\
\text { (2018). Fragilidade e } \\
\text { Desempenho } \\
\text { Cognitivo de Idosos } \\
\text { em Atendimento } \\
\text { Ambulatorial. Revista } \\
\text { Mineira de } \\
\text { Enfermagem, 22, e- } \\
\text { 1086. }\end{array}$ & $\begin{array}{l}\text { Trata-se de um estudo } \\
\text { transversal conduzido no } \\
\text { ambulatório de especialidades } \\
\text { de um hospital de ensino da } \\
\text { região dos Campos Gerais. } \\
\text { Foram selecionados } 374 \text { idosos } \\
\text { com } 60 \text { anos ou mais, com } \\
\text { pontuação superior ao ponto de } \\
\text { corte do Miniexame do Estado } \\
\text { Mental e estar aguardando } \\
\text { atendimento médico no dia da } \\
\text { entrevista. Caso o participante } \\
\text { tivesse alguma dificuldade de } \\
\text { comunicação verbal ou não } \\
\text { atingisse os pontos de corte, o } \\
\text { cuidador familiar era convidado } \\
\text { a participar da entrevista. }\end{array}$ & $\begin{array}{l}\text { Os resultados do estudo } \\
\text { destacaram a uma relação } \\
\text { estatística importante entre a } \\
\text { síndrome da fragilidade e } \\
\text { desempenho cognitivo em } \\
\text { pessoas idosas. Dessa forma, } \\
\text { sugere-se a busca precoce da } \\
\text { síndrome da fragilidade e do } \\
\text { declínio cognitivo, pois são } \\
\text { estados que interferem na } \\
\text { qualidade de vida da pessoa } \\
\text { idosa e colabora para a } \\
\text { mortalidade. }\end{array}$ & LILACS \\
\hline
\end{tabular}

Fonte: Autores (2020)

\section{Discussão}

Mediante os resultados obtidos na pesquisa, destacaram-se três categorias para um melhor entendimento da temática proposta. Os eixos temáticos foram organizados da seguinte maneira: O Estímulo Cognitivo e de Memória na Idosa sem Diagnóstico de Síndromes Demenciais e o Estímulo Cognitivo e de Memória na Idosa sem Diagnóstico de Síndromes Demenciais

Conforme a metodologia empregada, foi desenvolvida na categoria a Identificação do papel do enfermeiro no estímulo cognitivo e de memória em pessoas idosas, onde foi apresentada possíveis estratégias que podem ser implementadas para melhoria da cognição do idoso.

\subsection{O estímulo cognitivo e de memória na pessoa idosa sem diagnóstico de síndromes demenciais}

É destaque sobre a importância de programas e estratégias que realizem a manutenção da atividade cognitiva e de memória em pessoa idosa sem Diagnóstico de Síndromes Demenciais.

Segundo Yu et al (2009), a prática de atividade física regular, começando desde a meia-idade, pode influenciar na melhora do desempenho cognitivo na idade avançada. 
Contudo, mesmo com as atividades físicas e cognitivas de lazer estarem relacionadas com o atraso do início da demência, estudos futuros são necessários para aprimorar essa associação.

O exercício de treinamento de memória com atividades de repetição cognitiva tem resultados significativos no início das aulas, principalmente na primeira e na segunda experiência com tarefas cognitivas. Para McDougall Jr. et al (2010), os grupos de treinamento de memória alcançaram maiores ganhos na cognição global e obtiveram menores números de queixas de perdas de memória entre as pessoas idosas que faziam parte do estudo.

Segundo McDougall Jr. et al (2012), tornar saúde uma temática no qual as pessoas idosas se concentrem várias vezes por semana permite melhorar a capacidade de lidar com problemas de saúde, além da recuperação e processamento de informações através do uso de estratégias de memória.

É importante ressaltar a necessidade de pesquisas que investiguem maneiras mais eficazes de intervenções que melhorem a alfabetização em saúde para idosos como, por exemplo, através de atividades cognitivas e estratégias de memória que podem ser realizadas em oficinas coordenadas por um enfermeiro.

As atividades realizadas dentro do programa de integração de memória visual e motora, como conferir exercícios cognitivos que aumentem de dificuldade gradualmente, focando na melhoria cognitiva e de memória, possibilitam a neuroplasticidade, atividades recreativas e de apoio entre as pessoas idosas (Kim \& Suh, 2017).

De acordo com Kim \& Suh (2017), as estratégias específicas do programa de integração de memória visual e motora podem ser usadas para aprimorar a autoeficácia de memória de pessoas idosas, especialmente para aqueles com baixa autoeficácia. A integração visual-motora é a função cognitiva de maior importância para a vida diária do idoso independente, sendo assim é necessário a criação de novas abordagens englobando treinamentos de memória multifatorial.

\subsection{O estímulo cognitivo e de memória na pessoa idosa com diagnóstico de síndromes demenciais.}

Para pessoa idosa com Diagnóstico de Síndromes Demenciais, as publicações pesquisadas apresentaram propostas e estratégias para retardar e/ou prevenir o declínio cognitivo e de memória da pessoa idosa.

Induzir mudanças nas redes cerebrais com um programa de treinamento cognitivo e de memória é um método eficaz de estimulação para a pessoa idosa (Kim \& Suh, 2017). 
A aposentadoria pode provocar uma perda da percepção de propósito na vida, dando origem a um sentimento de afastamento da sociedade à medida que se envelhece e, consequentemente, levando a uma diminuição da qualidade de vida da pessoa idosa. Porém, acredita-se que a qualidade de vida da pessoa idosa com Diagnóstico de Síndromes Demenciais pode ser melhorada através de quatro fatores essenciais: estimulação cognitiva, prática de exercícios regulares, nutrição equilibrada e participação ativa na sociedade (Cruz et al, 2015).

De acordo com Kawashima (2013) evidenciou em um programa diário de treinamento contendo questões de literatura e problemas aritméticos para pessoas idosas com diagnóstico de demência tem um efeito benéfico imediato na velocidade de processamento mental. Além disso, esse sistema de treinamento de estimulação cognitiva mostra-se inteligente e acessível para a população idosa.

Segundo Cruz et al (2015), os cuidadores podem apresentar estratégias limitadas no cuidado com pessoa idosa com Diagnóstico de Síndromes Demenciais e impedir que muitas atividades de estimulação cognitiva sejam realizadas com mais frequência no domicílio. Por conta disso, a participação do enfermeiro torna-se crucial para a reorganização da dinâmica familiar, estimulando a participação dos membros da família nas oficinas e na vida desse idoso, permitindo que as atividades de estimulação não sejam prejudicadas.

Para Liang et al (2017), as consultas com equipe multidisciplinar, o manejo não farmacológico e os planos de cuidados mostraram fortes evidências da diminuição do declínio cognitivo para idosos com demência leve a moderada.

Segundo Silveira \& Silva (2020) parte-se do princípio de que, devido a necessidade excessiva de cuidados dispensadas ao paciente, os cuidadores passam a viver mudanças em seu cotidiano, privando-se dos momentos de lazer, e até momentos com a família, em consequência da função e necessidade de dedicação integral ao paciente com demência senil, acarretando em sobrecarga psicológica, física, emocional e social

Os medicamentos antidemenciais atualmente encontrados no mercado possuem uma eficácia limitada, por conta disso é fundamental que pesquisas futuras investiguem se as intervenções não farmacológicas trazem benefícios clínicos significativos para pessoa idosa com Diagnóstico de Síndromes Demenciais. 


\subsection{Identificação do papel do enfermeiro no estímulo cognitivo e de memória em pessoas idosas com e sem diagnósticos de síndromes demenciais}

Em relação à Identificação do papel do enfermeiro no estímulo cognitivo e de memória em pessoas idosas com e sem diagnósticos de síndrome demenciais, as publicações trazem diversos aspectos que destacam a importância e a autonomia do enfermeiro diante do estímulo cognitivo e de memória em pessoas idosas.

Segundo McDougall Jr. et al (2012) criação de uma variedade mais ampla de instrumentos para a melhoria a capacidade dos pesquisadores de verificar os estudos e desenvolver novos conhecimentos.

Ao realizar atividades de cognição e de memória com pessoas idosas, o enfermeiro pode aplicar novos instrumentos criados pelo mesmo, coletando informações que irão auxiliar para aprimorar as estratégias de cognição e de memória para esse público, além de gerar conteúdos pertinentes para as futures pesquisas que irão desenvolver novos conhecimentos e, consequentemente, melhorar a qualidade de vida da população idosa.

Kawashima (2013) propôs tarefas que incluem exercícios de leitura e atividades básicas sistematizadas em aritmética para pessoas idosas. A proposta de ler as atividades em voz alta permitiria a estimulação de vários processos cognitivos, por exemplo, o reconhecimento de palavras apresentadas visualmente, conversão dessas representações gráficas em representações fonológicas, análise dos significados das palavras e domínio da pronúncia. A solução das atividades em aritmética não poderia ser alcançada sem os processos cognitivos, por exemplo, o reconhecimento de números apresentados visualmente, execução de operações aritméticas e domínio do movimento das mãos.

As atividades de leitura e de aritmética são estratégias simples, fáceis e acessíveis para os idosos, com ou sem síndromes demenciais, podendo ser aplicadas pelo enfermeiro durante oficinas e/ou em casas de repouso. Esses exercícios irão promover a manutenção e a melhora das funções cognitivas e de memória de pessoas idosas, além da aproximação e confiança entre o enfermeiro e o idoso (Cruz et al, 2015).

Segundo Ibidem (2015) por conta das dificuldades enfrentadas pelos cuidadores de idosos como, por exemplo, desistência de outros empregos, sobrecarga de trabalho, alterações de humor e dificuldades financeiras, isso causaria limitações nas estratégias de cuidados ao idoso com demência e iria dificultar a realização de atividades de estimulação cognitiva e de memória dentro do ambiente domiciliar. Dessa forma, é de responsabilidade do enfermeiro 
instigar a família a participar também dos cuidado com o idoso para que não haja sobrecarga apenas para o cuidador e para que o idoso não sofra com um cuidado deficiente.

$\mathrm{O}$ enfermeiro tem o papel de orientar os cuidadores e familiares do idoso para que sejam realizadas atividades de estimulação cognitiva e de memória também no ambiente domiciliar e não somente nas oficinas, palestra e/ou encontros semanais com esses indivíduos. Essa estratégia permite uma técnica simples do cuidado de enfermagem em outros ambientes que o idoso convive, possibilitando a promoção da qualidade de vida da pessoa idosa e daqueles que são responsáveis pelo seu cuidado (Cruz et al, 2015).

A participação do enfermeiro dentro da equipe multidisciplinar é crucial, principalmente em intervenções não-farmacológicas, onde esse profissional de saúde pode propor diversas estratégias de estímulo cognitivo e de memória, como oficinas cognitivas, atividades de leitura e problemas matemáticos, jogos interativos utilizando o calendário e relógio e encontros semanais com esses idosos e seus respectivos cuidadores para avaliar como está o desenvolvimento cognitivo do idoso dentro do ambiente domiciliar (Cruz et al, 2015).

Kim \& Suh (2017) afirmam que o declínio da memória e da integração visual-motora causam diversos problemas na realização da maioria das atividades da vida diária do idoso como, por exemplo, relembrar nomes e palavras em uma conversa, lembrar o número do telefone, encontrar objetos, escrever e desenhar. Dessa forma, os idosos que enfrentam esse tipo de problema temem a perda da função cognitiva buscando maneiras de atrasar ou impedir esse avanço do declínio cognitivo.

Com essa busca de melhorar a inteligência cognitiva e a memória, de pessoas idosas, o enfermeiro deve ser capaz de atender essa demanda com estratégias de memória e integração visual-motora através de instrumentos com perguntas que irão somar pontos para analisar o declínio cognitivo desse indivíduo, além de outros exercícios como jogos de memória, ligação de pontos conforme as instruções, gestos rítmico com as mãos, etc. Após isso, realizar um feedback positivo e mostrar para a pessoa idosa o quanto ela evoluiu, durante esse programa, para que assim ela sinta-se encorajada e otimista para dar continuidade as atividades (Grden et al, 2018).

Para Grden et al (2018) é importante a análise de síndrome da fragilidade e escore cognitivo em pessoas idosas por instrumentos validados para a identificação precoce de fatores de risco, possibilitando aos enfermeiros e profissionais da saúde o planejamento de cuidados gerontológicos a essa população. 
A necessidade do enfermeiro de aplicar instrumentos validados se mostra presente quando se busca novos conhecimentos e estratégias para melhorar as intervenções de estímulo cognitivo e de memória em idosos que apresentem fragilidades. Dessa forma, com novas informações adquiridas através das aplicações dos instrumentos, o enfermeiro tem a possibilidade de organizar novas medidas que irão melhorar a qualidade de vida desse sujeito.

\section{Considerações Finais}

Com análise das publicações pesquisadas nas bases de dados científicas, é visível a importância do enfermeiro no estímulo cognitivo e de memória em pessoas idosas, pois através de oficinas, estratégias e/ou treinamentos, que são competências do enfermeiro, podese melhorar a cognição e memória de pessoas idosas e atrasando o avanço de síndrome demenciais e, consequentemente, trazendo uma melhor qualidade de vida para esse público.

Durante a análise dos estudos perceberam-se diversos métodos de estimulação cognitiva e de memória que podem ser postos em prática pelo enfermeiro e o quanto a sua participação dentro de uma equipe multidisciplinar tem destaque quando se trata de estratégias para exercitar a cognição e memória da pessoa idosa, além de buscar meios não farmacológicos de retardar o surgimento de síndromes demências, principalmente a Doença de Alzheimer.

Durante a busca nas bases de dados científicas notou-se um número limitado de publicações acerca do tema abordado no presente estudo. Diversas pesquisas encontradas estavam voltadas para outros profissionais de saúde, e raramente citando o papel do enfermeiro no estímulo cognitivo e de memória em pessoas idosas.

Recomenda-se que pesquisas futuras sobre o tema busquem novas estratégias e tecnologias de competências do profissional enfermeiro para a manutenção da inteligência cognitiva e da memória em idosos saudáveis e retardando o avanço das síndromes demenciais, principalmente a Doença de Alzheimer.

\section{Referências}

Cruz, TJP, Sá, SPC, Lindolpho, MC \& Caldas, CP. (2015). Estimulação cognitiva para idoso com Doença de Alzheimer realizada pelo cuidador. Revista Brasileira de Enfermagem, 68(4), 510-516. 
Delavande, A, Hurd, MD, Martorell, P \& Langa, KM. (2013). Dementia and out-of-pocket spending on health care services. Alzheimers Dement, 9(1), 19-29.

Ferreira, OGL, Maciel, SC, Costa, SMG, Silva, AO \& Moreira, MASP (2012).

Envelhecimento ativo e sua relação com a independência funcional. Texto Contexto Enferm, 21(3), 513-518.

Grden, CRB, Ivastcheschen, T, Cabral, LPA, Reche, PM, Rodrigues, CRB \& Borges, PKO. (2018). Fragilidade e Desempenho Cognitivo de Idosos em Atendimento Ambulatorial. Revista Mineira de Enfermagem, 22, e-1086.

Instituto Brasileiro de Geografia e Estatística (2018). Pesquisa Nacional por Amostra de Domicílios Contínua de 2018. IBGE, Recuperado em 22 de Abril, 2020, https://ww2.ibge.gov.br/home/estatistica/pesquisas/pesquisa_resultados.php?id_pesquisa=149

Kawashima, R. (2013). Mental Exercises For Cognitive Function: Clinical Evidence. Journal of Preventive Medicine and Public Health, 46(1), 22-27.

Kim, E \& Suh, S. (2017). Effects of a Memory and Visual-Motor Integration Program for Older Adults Based on Self-Efficacy Theory. Journal of Korean Academy of Nursing, 47(3), 431-444.

Liang, C, Chou, M, Chen, L, Wang, K, Lin, S, Chen, L, Lin, Y, Liu, T \& Loh, C. (2017). Delaying cognitive and physical decline through multidomain interventions for residentes with mild-to-moderate dementia in dementia care units in Taiwan: A prospective cohort study. Geriatr. Gerontol. Int, 17(1), 36-43.

Mcdougall, JR, Graham J, Mackert, M \& Becker, H. (2012). Memory Performance, Health Literacy, and Instrumental Activities of Daily Living of Community Residing Older Adults. Nursing Res, 61(1), 70-75. 
McDougall, GJ Jr., Becker, H, Pituch, K, Acee, TW, Vaughan, PW, Delville, CL. (2010). The SeniorWISE study: improving everyday memory in older adults. Arch Psychiatr Nurs, 24(5), 291-306.

Ministério da Saúde (2018). Agenda nacional de prioridades de pesquisa em saúde. MS, Recuperado em 30 de Abril, 2020, https://bvsms.saude.gov.br/bvs/publicacoes/agenda_prioridades_pesquisa_ms.pdf

Morando, EMG, Schmitt, JC \& Ferreira, MEC. (2017). Envelhecimento, Autocuidado e Memória: Intervenção como Estratégia de Prevenção. Revista Kairós Gerontologia, 20(2), 353-374.

Organização Mundial da Saúde (2014). "Ageing well” must be a global priority. OMS, Recuperado em 30 de Abril, 2020, http://www.who.int/mediacentre/news/releases/2014/lancet-ageing-series/en/>

Silveira, AG \& Silva, DA. (2020). Sobrecarga dos familiares no cuidado ao portador de demência senil: uma revisão integrativa. Research, Society and Development, 9(6), e179963671.

Soares, CB, Hoga, LAK, Peduzzi, M, Sangaleti, C, Yonekura, T \& Silva, DRA. D. (2014). Revisão Integrativa: conceitos e métodos utilizados na enfermagem. Rev. Esc. Enfermagem USP, 48(2), 335-345.

Souza, MT, Silva, MD \& Carvalho, R. (2010). Revisão Integrativa: O que é e como fazer. Einstein, 8(1), 102-106.

Yu, F, Ryan, LH, Schaie, KW, Willis, SL, \& Kolanowski, A. (2009). Factors Associated With Cognition in Adults: The Seattle Longitudinal Study. Res. Nurs. Health, 32(5), 540-550. 
Research, Society and Development, v. 9, n. 7, e306974180, 2020

(CC BY 4.0) | ISSN 2525-3409 | DOI: http://dx.doi.org/10.33448/rsd-v9i7.4180

Porcentagem de contribuição de cada autor no manuscrito

Lucas de Almeida Abrahão - 60\%

Alessandra Conceição Leite Funchal Camacho - 40\% 